\title{
Acupuncture improves white matter perfusion and white matter integrity in vascular dementia rats: an MRI-based imaging study
}

Si-Ming Ma ${ }^{1}$, Lu Wang ${ }^{2}$, Xin-Tong $\mathrm{Su}^{2}$, Jin Huang ${ }^{1}$, Lu-Lu Lin ${ }^{2}$, Jia-Kai Shao ${ }^{1}$, Jing-Wen Yang ${ }^{2 *}$, Cun-Zhi Liu ${ }^{2}$

1. Beijing Hospital of Traditional Chinese Medicine affiliated to Capital Medical University, Beijing, China

2. School of Acupuncture-Moxibustion and Tuina, Beijing University of Chinese Medicine, Beijing, China

\section{Correspondence:}

Jing-Wen Yang, School of Acupuncture-Moxibustion and Tuina, Beijing University of Chinese Medicine, 11 Beisanhuan East Road, Chaoyang District, Beijing 100029, China

E-mail: yangjw0626@126.com

\begin{abstract}
Background: White matter lesions induced by chronic cerebral hypoperfusion $(\mathrm{CCH})$ are common pathological changes, and are associated with cognitive impairment in vascular dementia $(\mathrm{VaD})$. It has been reported that acupuncture can improve cognitive deficits of VaD rats through increasing cortical cerebral blood flow and attenuating neuroinflammation. However, the effects of acupuncture on white matter perfusion and white matter structural integrity have been not investigated.
\end{abstract}

Methods: VaD was induced by bilateral common carotid arteries occlusion (BCCAO) in Wistar rats. Morris water maze (MWM) was used to evaluated the spatial learning and memory of rats. Arterial spin labeling imaging (ASL) and diffusion tensor imaging (DTI) were performed to measure the cerebral blood flow and white matter integrity in corpus callosum, external capsule, internal capsule, optic nerve and optic tract. Pathological staining was also applied to detect the myelin loss and neuroinflammation.

Results: BCCAO rats with declined cerebral blood flow exhibited significant worse MWM performance, and altered DTI parameters including decreased fractional anisotropy, elevated radial diffusivity and axial diffusivity in white matter regions. After acupuncture treatment at GV20 and ST36, cognitive dysfunction, disruption of white matter perfusion and integrity were reversed. Pathological results supported that 
acupuncture could attenuate the loss of myelin sheath and microglia activation.

Conclusions: Our findings suggest that acupuncture treatment protects cognitive impairment of BCCAO rats through increasing subcortical white matter perfusion and improving white matter lesions.

Keywords: Acupuncture; ASL; DTI; cerebral blood flow; white matter; microglia activation

\section{INTRODUCTION}

Vascular dementia (VaD), induced by risk factors of cardiovascular or cerebral vascular system [1], is the second most common dementia type only to Alzheimer's disease [2]. Epidemiological survey demonstrated that the rate of prevalence is strongly associated with aging [3], with the risk of doubling every 5.3 years $[1,4]$. $\mathrm{VaD}$ is also characterized as cerebrovascular disorder, which incorporates hemodynamics alteration including persistent cerebral blood flow (CBF) attenuation and dysfunction of $\mathrm{CBF}$ regulation $[5,6]$. These circulation disruptions are mainly attribute to the aging vasculature failing to cope with biological demands, and result in subcortical ischemia and white matter lesions [7]. Ample evidences suggest that chronic cerebral hypoperfusion $(\mathrm{CCH})$ is a leading pathophysiological mechanism of white matter lesions in $\mathrm{VaD}$ [4]. Cerebral hypoxia-ischemia induced by $\mathrm{CCH}$ is sufficient to trigger the white matter inflammation and oxidative stress [8,9], which, in turn, impacts the regulation of cerebral blood supply [4, 10]. Such imbalance of CBF regulation would aggravate the white matter injury and contribute to the loss of working memory or execution dysfunction $[11,12]$.

White matter is a subcortical structure that consists of neuronal axons, myelin sheath and the surrounding glia cells. Due to the deep location at the nonoverlapping area of cerebral arterioles or capillary, white matter is vulnerable to the declined CBF [13]. White matter lesions caused by ischemia are common pathological changes in $\mathrm{VaD}$, encompasses axons damage, loss of myelin associated glycoproteins and microglia activation [14-16]. The diffusion tensor imaging (DTI) evidences proved that $\mathrm{VaD}$ individuals showed a specific pattern of white matter lesions more affected in thalamic radiations or corpus callosum (CC) [17, 18], appeared as reduced fractional anisotropy (FA), elevated radial diffusivity (RD) and axial diffusivity (AD) $[19,20]$. Arterial spin labeling (ASL) imaging study supported that subjects suffering 
from $\mathrm{VaD}$ develop white matter lesions being concomitant with persistent reduction of CBF [21]. This parenchymal CBF would remain decreased at $50 \%$ of the basic level, and at least last two weeks [22].

Acupuncture is a nonpharmacologic therapy of traditional Chinese medicine originated from ancient's clinical practice, and has been operated to stimulate points or meridian which spread over the surface of body and within many organs [23]. Clinical studies confirm that $\mathrm{VaD}$ patients treated with acupuncture showed amelioration of cognitive status (memory, orientation, calculation and self-managing ability), where the increased cortical CBF might underlie the therapeutic effects [24-26]. Animal experiments further reveal that the beneficial effect of acupuncture on $\mathrm{CBF}$ is associated with promotion of cholinergic vasodilative system or suppression of oxidative stress in cortex [26, 27]. These researches have investigated the role of acupuncture in cortical blood flow. Nevertheless, the effects of acupuncture on white matter perfusion and structural integrity have not been observed. In this study, we used ASL to monitor CBF changes of CC, external capsule (EC), internal capsule (IC), optic tract (OT), and optic nerves (ON) in rats after bilateral common carotid arteries occlusion (BCCAO) and acupuncture treatment. White matter lesions were also observed by DTI so as to evaluate the disruption of white matter integrity. Additionally, pathological changes of myelin and glia in white matter were detected to validate the features of myelin loss and inflammatory response.

\section{MATERIAL AND METHODS}

\subsection{Animals}

Thirty two male Wistar rats (260-280g) were housed on 12/12 h light/dark cycle with controlled temperature $\left(24 \pm 0.5^{\circ} \mathrm{C}\right)$ in the animal facility at Dongfang hospital, Beijing University of Chinese Medicine. Food and water were available ad libitum for rats. Rats were randomized into four groups by digital table: sham-operated rats (SHAM), rats subjected to BCCAO (BCCAO), BCCAO rats treated with acupuncture $(\mathrm{ACU})$ and BCCAO rats treated with non-acupoints (NON-ACU). All experiments compliant with the procedures of the US National Institutes of Health Guide for the Care and Use of Laboratory Animals, and were approved by the Instituional Animal Care and Use Committee of Dongfang hospital, Beijing University of Chinese Medicine. 


\subsection{Surgical procedures}

VaD model was induced by BCCAO method as preciously described [28]. Rats were anesthetized with intraperitoneal injection of sodium pentobarbital $(40 \mathrm{mg} / \mathrm{kg}$ body weight). A ventral cervical midline incision was made, and both common carotid arteries were exposed and permanently double ligated with 4-0 silk sutures. In the SHAM group, the same operation was manipulated as in the BCCAO group except for artery occlusion.

\subsection{Acupuncture treatment}

Three days after surgery, rats received a two-week acupuncture therapy, once daily and one-day rest after six treatments. Acupuncture needles (Hwato, China, $0.22 \times 5 \mathrm{~mm}$ ) were used for stimulation. In ACU group, treatment was performed at "Baihui" (GV20) (located at midline of the head, approximately midway on the line connecting the apices of the auricles) and bilateral "Zusanli" (ST36) (located at 2mm lateral to the anterior tubercle of the tibia, and $5 \mathrm{~mm}$ below the capitulum fibulae under the knee joint) acupoints. For NON-ACU group, bilateral non-acupoints (located at $10 \mathrm{~mm}$ upper to the anterior superior spine) were chosen as the insertion site. The needles were retained for $10 \mathrm{~min}$ before removal. All the other non-treatment groups were performed with the same catching-grasping stimulation as the ACU group.

\subsection{Morris Water Maze Test}

The Morris Water Maze (MWM) test consists of a circular pool $160 \mathrm{~cm}$ in diameter and was divided into 4 quadrants. During the training, all rats were subjected to 3 training trials per day for 5 consecutive days. In each trial, animals were allowed $90 \mathrm{~s}$ to search the submerged platform and $10 \mathrm{~s}$ to remain on it. The latency to escape and swimming speed were recorded. On day 6 , the platform was removed for conducting probe trial, and the rats were permitted to swim freely for $90 \mathrm{~s}$. The time spent in target quadrant was recorded. The experimental procedure is shown in Figure 1.

\subsection{MRI acquisition}

Rats were induced anesthesia and maintained in mixture of $2 \%$ isoflurane and $98 \%$ oxygen during scanning. All images were acquired with a 7.0 T MR scanner (Bruker BioSpin GmbH, Rheinstetten, Germany). Coronal $\mathrm{T}_{2} \mathrm{WI}$ rapid acquisition with relaxation enhancement (RARE) was performed with the following parameters: repetition time $(\mathrm{TR})=5000 \mathrm{~ms}$; echo time $(\mathrm{TE})=36 \mathrm{~ms}$; flip angle $=180^{\circ}$; slice thickness $=0.7 \mathrm{~mm}$; field-of-view $(\mathrm{FOV})=3.5 \times 3.5 \mathrm{~cm} ;$ matrix $=256 \times 256$. Perfusion 
images were captured using the continuous ASL with echo-planar imaging fluid-attenuated inversion recovery (EPI-FLAIR) sequence, and the parameters are: $\mathrm{TR}=18000 \mathrm{~ms} ; \mathrm{TE}=25 \mathrm{~ms}$; flip angle $=90^{\circ}$; slice thickness $=2 \mathrm{~mm}, \mathrm{FOV}=3.0 \times 3.0 \mathrm{~cm}$, matrix $=128 \times 128$. Acquisition of DTI using spin echo planner imaging (SE) sequence was set by $\mathrm{TR}=6250 \mathrm{~ms}$; $\mathrm{TE}=22 \mathrm{~ms}$; flip angle $=90^{\circ}$; slice thickness $=0.8 \mathrm{~mm}$; $\mathrm{FOV}=3.0 \times 3.0 \mathrm{~cm}$; matrix $=128 \times 128, \mathrm{~b}$ values $=0$ and $1000 \mathrm{~s} / \mathrm{mm}^{2}$ along with a total of 30 diffusion gradient directions.

\subsection{Data processing}

\subsubsection{CBF and DTI parameters calculation}

ASL images were reconstructed automatically with paravision v5.1 software (Bruker, Pharmascan, Germany). The CC and EC were drawn manually according to T2WI images to measure the CBF.

DTI raw data was processed using the DSI Studio Software (http://dsi-studio.labsolver.org). Files of 2Dseq were loaded directly into software for distortion correction. A b-table was added to flip $\mathrm{x}, \mathrm{y}$ or $\mathrm{z}$ coordinate, and eddy correction was conducted for the calibration of gradient magnetic field. For the reconstruction part, a mask was generated using DTI method to filtered out the background region [29]. The CC, EC, internal capsule (IC), optic tract (OT) and optic nerves $(\mathrm{ON})$ were selected as regions of interest (ROIs) drawn manually according to Paxinos rat brain atlas (Figure 4A) [30]. DTI parameters including fractional anisotropy (FA), axial diffusivity (AD), radial diffusivity (RD), and mean diffusivity (MD) were calculated. All imaging data was processed by one independent analyst.

\subsubsection{White matter fiber tracking}

Fiber tracking was conducted to depict the fiber numbers and length in 4 groups. Fiber tracts were seeded from $\mathrm{CC}$ and EC in each rat. The tracking parameters was set as follow: tracking index: FA, angular threshold: $45^{\circ}$; step size: $0.05 \mathrm{~mm}$; smoothing: 0.01; min length: $0.1 \mathrm{~mm}$; max length: $30 \mathrm{~mm}$; seed orientation: Primary. The fiber density (fiber number/ voxel number) and fiber length were calculated according to the previous study [20].

\subsection{Immunohistochemistry}

After the behavioral text, all rats were injected with an overdose sodium pentobarbital $(100 \mathrm{mg} / \mathrm{kg})$. The brain tissues of rats were collected and embedded in optimal cutting temperature compound. Brain frozen tissues were sectioned with a 
freezing microtome to a thickness of $10 \mu \mathrm{m}$. Brain sections were washed by PBS, blocked with $3 \%$ hydroxide and incubated with primary antibodies to myelin basic protein (MBP, 1:100, Abcam), ionized calcium binding adapter molecule1 (Iba-1, 1:1000, Abcam) and glial fibrillary acidic protein (GFAP, 1:1000, Abcam) at $4^{\circ} \mathrm{C}$ overnight. Subsequently, the sections were incubated with biotinylated secondary antibodies (1:100, ZSGB-BIO) and 3,3' diaminobenzadine tetrahydrochloride (DAB) as a chromogenic substrate (Solarbio). DAB immunolabeled sections were captured using an optical microscope (Nikon DS-Ri2). All measurements were analyzed blindly by a researcher using ImageJ v2.0.0 software.

\subsection{Statistical analysis}

Statistical analysis was performed using SPSS v22.0 software. Graphs were generated by Graphpad Prism 8 software. Results of Morris water maze were analyzed by two-way repeated measures ANOVA. CBF, DTI parameters, fiber numbers, fiber length, MBP density, Iba-1 number and GFAP number were compared using one-way ANOVA followed by post hoc Tukey's test. Correlation analysis between $\mathrm{CBF}$ and FA in SHAM and ACU group were conducted with two-tail Pearson's correlation. All data are presented as mean \pm standard error of mean (SEM), and $\mathrm{p}<0.05$ was set statistically significant.

\section{RESULTS}

\subsection{Effects of acupuncture on cognitive impairments in VaD rats}

We used MWM to examine spatial learning and memory to observe the effects of acupuncture on cognitive impairments. In BCCAO group, rats spent more time on finding the hidden platform than SHAM group in the MWM test (Figure 2A). The rats treated with acupuncture at ST36 and GV20 showed better escape latency than BCCAO rats from day 2 of the probe trial (compared with BCCAO group, $\mathrm{p}<0.01$ ), and acupuncture at non-acupoint did not improve the performance. For the time spend in target quadrant (Figure 2B), there was a markedly decrease in BCCAO group (compared with SHAM group, $\mathrm{p}<0.0001$ ). Acupuncture at GV20 and ST36 significant increased the time that rats spent in the target quadrant (compared with BCCAO group, $\mathrm{p}<0.01$ ), but no significant difference was found between NON-ACU group and BCCAO group $(p=0.9871)$. Among the four groups, there was no significant difference in swimming speed (Figure 2C), suggesting the unaffected 
motor function.

\subsection{Effects of acupuncture on the white matter CBF}

The location of ROIs including CC and EC was marked at brain heatmaps (Figure 3A). As shown in Figure 3B, $\mathrm{CBF}$ in $\mathrm{CC}$ and $\mathrm{EC}$ is significant decreased to 13.159 $\mathrm{ml} / 100 \mathrm{~g} / \mathrm{min}$ in BCCAO rats $(\mathrm{p}<0.01$, compared with $20.713 \mathrm{ml} / 100 \mathrm{~g} / \mathrm{min}$ in SHAM group). However, in ACU group, the white matter perfusion improved to 20.209 $\mathrm{ml} / 100 \mathrm{~g} / \mathrm{min}$ ( $\mathrm{p}<0.01$, compared with BCCAO group). Furthermore, there was no significant difference between BCCAO and NON-ACU group ( $p=0.5177, \mathrm{CBF}$ of NON-ACU: $14.699 \mathrm{ml} / 100 \mathrm{~g} / \mathrm{min}$ ), which means acupuncture at non-acupoint did not protect the white matter from $\mathrm{CCH}$.

\subsection{Effects of acupuncture on white matter integrity in DTI}

$\mathrm{FA}, \mathrm{RD}, \mathrm{AD}$, and $\mathrm{MD}$ values in $\mathrm{CC}, \mathrm{EC}, \mathrm{IC}, \mathrm{ON}$, and $\mathrm{OT}$ were calculated using DSI studio (Figure 4A). As shown in figure 4B, significant decrease of FA and increase of $\mathrm{RD}$ in all representative ROIs were observed in BCCAO rats in comparison to SHAM group (CC: $p<0.0001$, EC: $p<0.0001$, IC: $p<0.05$, ON: $p<$ 0.0001 , OT: $p<0.0001$ ), suggesting an extensive damage of white matter integrity. After a two-week acupuncture treatment, the aggravated tendency of FA and RD induced by BCCAO was reversed (except RD value in ON). In NON-ACU group, non-acupoint treatment did not rescue the effects of BCCAO surgery on FA and RD in all ROIs. Statistical differences of AD in CC $(p<0.01)$ and MD in OT $(p<0.01)$ were also observed between SHAM and BCCAO group. But these two DTI indices were not affected by acupuncture. Similarly, Results of fiber tracking demonstrated that a prominent reduction of fiber density induced by BCCAO was attenuated by acupuncture $(p<0.01)$, while the fiber length was not affected by surgery or acupuncture treatment. Besides, Pearson's correlation analysis revealed that $\mathrm{CBF}$ was positive correlated with FA values $(r=0.9395, r=0.9428$, respectively) and white matter fiber density ( $\mathrm{r}=0.9756, \mathrm{r}=0.9712$, respectively), and negative correlated with $\mathrm{RD}$ values $(\mathrm{r}=0.9711, \mathrm{r}=0.9537$, respectively) in SHAM and ACU group (Figure 4C).

\subsection{Effects of acupuncture on white matter pathology}

To evaluate the pathological changes of all types of glia in white matter region, we examined the myelin basic protein (MBP, located at membrane of oligodendrocyte), Iba-1 and GFAP expression (Figure 6). We found that MBP was reduced significantly 
at the 14th day after BCCAO surgery (compared with SHAM group, $\mathrm{p}<0.001$ ), while acupuncture treatment alleviated the MBP loss effectively in CC (compared with BCCAO group, $\mathrm{p}<0.01)$. Synchronously, the number of Iba-1 positive microglia in $\mathrm{CC}$ was increased significantly in BCCAO group (compared with SHAM group, $\mathrm{p}<$ 0.001), and decreased in ACU group (compared with BCCAO group, $\mathrm{p}<0.001$ ), indicating that the prominent neural inflammation in white matter was reduced by acupuncture at GV20 and ST36 but not non-acupoint. There was no remarkable variance of GFAP-positive astrocyte number among the four groups.

\section{DISCUSSION}

In current study, the MWM results suggest that spatial learning and memory in BCCAO rats was improved by acupuncture. Since these cognitive impairment performance are associated with white matter lesions in $\mathrm{CCH}[20,31]$, we used MRI-based ASL and DTI to evaluate the white matter perfusion and the white matter microstructure after BCCAO and acupuncture for the first time. In $\mathrm{CC}$, where the white matter fibers are the most abundant, the loss of MBP was protected by acupuncture at GV20 and ST36. In addition, we found significant microglia activation in white matter regions was attenuated after acupuncture treatment. This pathological change furtherly profiled the protective effect of acupuncture on white matter in BCCAO-induced VaD rats.

Large number of studies suggested that spatial learning and memory are impaired in BCCAO rats. As a peripheral stimulation, acupuncture could improve the performance of dementia rodents in MWM by elevating the CBF velocity and volume of prefrontal lobe and hippocampus [32, 33]. In this study, GV20 and ST36 were selected based on our published results as an ideal combination of acupoints that is benefit on memory deficits in BCCAO-induced $\mathrm{VaD}$ rats $[34,35]$. Consistent with previous studies, we found that BCCAO rats with increased escape latency and decreased time in target quadrant in probe trial showed declined white matter CBF. In contrast, VaD rats treated with two-week acupuncture had better MWM performance and white matter perfusion. These outcomes indicate that acupuncture can improve cognitive dysfunction of $\mathrm{VaD}$ rats by increasing the white matter $\mathrm{CBF}$.

DTI is a sensitive MRI technique that could highlight the changes of white matter microstructure in term of diffusion properties of tissue in vivo [17]. FA and MD 
represent the white matter organization in general, while $\mathrm{RD}$ and $\mathrm{AD}$ reflect quality of the myelin sheath and the axon integrity, respectively [36]. Human study indicated that subcortical $\mathrm{VaD}$ patients had decreased FA and increased MD in all white matter regions except occipital areas [37]. But the DTI parameters reported in experimental $\mathrm{VaD}$ rats showed more heterogeneous, characterized by remarkable alteration of FA, $\mathrm{RD}$ and $\mathrm{AD}$ in $\mathrm{ON}$, OT and EC 2 weeks after BCCAO, or significant reduces of MD, $\mathrm{RD}$ and $\mathrm{AD}$ in $\mathrm{CC}$ and EC 5 weeks after surgery [20,38]. We combined ASL with DTI and found that predominant reduction of CBF volume correlated with significant white matter disintegration at 2 weeks after surgery, appeared as lower FA and higher $\mathrm{RD}$ values in white matter regions, which was in line with previous study. After acupuncture treatment, FA, RD and white matter perfusion were significantly ameliorated. Therefore, we conclude that compromised myelin sheath (represents oligodendrocyte) but not neuroaxonal damage was rescued by acupuncture.

Visualizing results furtherly explained the specific changes of DTI parameters. In this part, we produced quantitative analysis of white matter based on previous study, which suggested the remarkable decreased fiber density and fiber length 4 weeks after BCCAO [20]. However, we only observed contrasting changes in fiber density but not fiber length in four groups, indicating that the wrapped myelin sheath was damaged by ischemia, but neuroaxon structure was still intact. Given that our MRI acquisition were arranged at the 13th day after surgery, we speculate that the axon had not been attacked by ischemia. Taken together, our diffusion imaging outcomes proved partially that acupuncture treatment protects myelin sheath integrity of subcortical white matter from $\mathrm{CCH}$ in $\mathrm{VaD}$ rats.

We also observed the specific pathological changes of white matter to evaluate the demyelination and neuroinflammation. In alignment with the DTI results, we found that the loss of MBP expression and the increase of Iba-1 positive cells were prominent in rats with $\mathrm{BCCAO}$, and a two-week acupuncture treatment halted the pathological process. These findings suggest that the disintegration of white matter characterized by loss of myelin sheath and microglia activation could be attenuated by acupuncture. It is worth noting that astrogliosis was not significant in our study. According to the previous work, both the astrocyte and microglia contribute to the neuroinflammation and white matter lesions in $\mathrm{VaD}$ rats [39]. This might attribute to the different chronic hypoperfusion model: bilateral common carotid artery stenosis 
that used in that experiment. Although we did not observe the astrocyte activation, our study does not rule out the possibility that astrocyte plays crucial role in protective effects of acupuncture in white matter lesions. Therefore, the further work needs to be done in order to elucidate the glia-associated neural circuits that acupuncture could regulate in $\mathrm{VaD}$ rats.

\section{CONCLUSIONS}

In present study, we used neuroimaging and immunohistochemistry technique to investigate the effects of acupuncture on cognitive dysfunction of $\mathrm{CCH}$-induced $\mathrm{VaD}$ rats. Our results reveal that acupuncture protects spatial and learning memory of $\mathrm{BCCAO}$ rats through increasing subcortical white matter perfusion, improving white matter integrity and attenuating myelin loss. Intensive studies of down-stream neural circuits in $\mathrm{VaD}$ are encouraged to confirm the efficacy of acupuncture in $\mathrm{VaD}$.

\section{ABBREVIATIONS}

$\mathrm{CCH}$ : chronic cerebral hypoperfusion; VaD: vascular dementia; BCCAO: bilateral common carotid arteries occlusion; MWM: Morris water maze; ASL: Arterial spin labeling; DTI: diffusion tensor imaging; CBF: cerebral blood flow; ROIs: regions of interest; CC: corpus callosum; EC: external capsule; IC: internal capsule; ON: optic nerve; OT: optic tract; FA: fractional anisotropy; RD: radial diffusivity; AD: axial diffusivity; MD: mean diffusivity; MBP: myelin basic protein; Iba-1: ionized calcium binding adapter molecule 1, GFAP: glial fibrillary acidic protein.

\section{DECLARATIONS}

\section{Ethics approval}

All experiments were approved by the Instituional Animal Care and Use Committee of the Dongfang hospital, Beijing University of Chinese Medicine (ethics approval number: 201822).

\section{Consent for publication}

Not applicable

\section{Availability of data and materials}

The data used to support the findings of this study are available from the corresponding authors upon request. 


\section{Competing interests}

The authors declare that they have no competing interests.

\section{Funding}

This work was funded by grants from the National Natural Science Foundation of China (81774396).

\section{Authors' contributions}

MSM performed imaging processing, pathological staining and wrote the manuscript. LW and XTS established the animal model, and performed behavioral test. JH and LLL analyzed data of behavioral test and pathological staining. JKS participated in the ASL imaging and partial DTI imaging processing. CZL and JWY were responsible for designing and supervising the study. All authors critically reviewed and approved the manuscript.

\section{Acknowledgments}

We would like to thank Mr. Jian-Feng Lei and Mr. Zhan-Jing Wang for the instruction of MRI imaging scan.

\section{REFERENCES}

1. Dichgans M, Leys D: Vascular Cognitive Impairment. Circulation Research 2017, 120:573-591.

2. Gorelick PB, Scuteri A, Black SE, DeCarli C, Greenberg SM, Iadecola C, Launer LJ, Laurent S, Lopez OL, Nyenhuis D, et al: Vascular Contributions to Cognitive Impairment and Dementia A Statement for Healthcare Professionals From the American Heart Association/American Stroke Association. Stroke 2011, 42:2672-2713.

3. Jellinger KA: Morphologic diagnosis of "vascular dementia" - A critical update. Journal of the Neurological Sciences 2008, 270:1-12.

4. Iadecola C: The Pathobiology of Vascular Dementia. Neuron 2013, 80:844-866.

5. Ogoh S: Relationship between cognitive function and regulation of cerebral blood flow. Journal of Physiological Sciences 2017, 67:345-351.

6. Giezendanner S, Fisler MS, Soravia LM, Andreotti J, Walther S, Wiest R, 
Dierks T, Federspiel A: Microstructure and Cerebral Blood Flow within White Matter of the Human Brain: A TBSS Analysis. Plos One 2016, 11.

7. Alber J, Alladi S, Bae HJ, Barton DA, Beckett LA, Bell JM, Berman SE, Biessels GJ, Black SE, Bos I, et al: White matter hyperintensities in vascular contributions to cognitive impairment and dementia (VCID): Knowledge gaps and opportunities. Alzheimers Dement (N Y) 2019, 5:107-117.

8. Dong Y-F, Kataoka K, Toyama K, Sueta D, Koibuchi N, Yamamoto E, Yata K, Tomimoto H, Ogawa H, Kim-Mitsuyama S: Attenuation of brain damage and cognitive impairment by direct renin inhibition in mice with chronic cerebral hypoperfusion. Hypertension 2011, 58:635-642.

9. Miyamoto N, Maki T, Pham LD, Hayakawa K, Seo JH, Mandeville ET, Mandeville JB, Kim KW, Lo EH, Arai K: Oxidative stress interferes with white matter renewal after prolonged cerebral hypoperfusion in mice. Stroke 2013, 44:3516-3521.

10. Faraci FM: Protecting against vascular disease in brain. Am J Physiol Heart Circ Physiol 2011, 300:H1566-1582.

11. Calabrese V, Giordano J, Signorile A, Ontario ML, Castorina S, De Pasquale C, Eckert G, Calabrese EJ: Major Pathogenic Mechanisms in Vascular Dementia: Roles of Cellular Stress Response and Hormesis in Neuroprotection. Journal of Neuroscience Research 2016, 94:1588-1603.

12. Du S-Q, Wang X-R, Xiao L-Y, Tu J-F, Zhu W, He T, Liu C-Z: Molecular Mechanisms of Vascular Dementia: What Can Be Learned from Animal Models of Chronic Cerebral Hypoperfusion? Molecular Neurobiology 2017, 54:3670-3682.

13. Li B, Ohtomo R, Thunemann M, Adams SR, Yang J, Fu B, Yaseen MA, Ran C, Polimeni JR, Boas DA, et al: Two-photon microscopic imaging of capillary red blood cell flux in mouse brain reveals vulnerability of cerebral white matter to hypoperfusion. J Cereb Blood Flow Metab 2020, 40:501-512.

14. Struys T, Govaerts K, Oosterlinck W, Casteels C, Bronckaers A, Koole M, Van Laere K, Herijgers P, Lambrichts I, Himmelreich U, Dresselaers T: In vivo evidence for long-term vascular remodeling resulting from chronic cerebral hypoperfusion in mice. Journal of Cerebral Blood Flow and Metabolism 2017, 37:726-739. 
15. Zhang L-Y, Pan J, Mamtilahun M, Zhu Y, Wang L, Venkatesh A, Shi R, Tu X, Jin K, Wang Y, et al: Microglia exacerbate white matter injury via complement C3/C3aR pathway after hypoperfusion. Theranostics 2020, 10:74-90.

16. Qin C, Liu Q, Hu Z-W, Zhou L-Q, Shang K, Bosco DB, Wu -LJ, Tian D-S, Wang W: Microglial TLR4-dependent autophagy induces ischemic white matter damage via STAT1/6 pathway. Theranostics 2018, 8:5434-5451.

17. Palesi F, De Rinaldis A, Vitali P, Castellazzi G, Casiraghi L, Germani G, Bernini S, Anzalone N, Ramusino MC, Denaro FM, et al: Specific Patterns of White Matter Alterations Help Distinguishing Alzheimer's and Vascular Dementia. Front Neurosci 2018, 25(12):274.

18. Fu J-L, Zhang T, Chang C, Zhang Y-Z, Li W-B: The value of diffusion tensor imaging in the differential diagnosis of subcortical ischemic vascular dementia and Alzheimer's disease in patients with only mild white matter alterations on T2-weighted images. Acta Radiologica 2012, 53:312-317.

19. Ben-Ari H, Lifschyz T, Wolf G, Rigbi A, Blumenfeld-Katzir T, Merzel TK, Koroukhov N, Lotan A, Lerer B: White matter lesions, cerebral inflammation and cognitive function in a mouse model of cerebral hypoperfusion. Brain Research 2019, 1711:193-201.

20. Choi B-R, Kim D-H, Bin Back D, Kang CH, Moon W-J, Han J-S, Choi D-H, Kwon KJ, Shin CY, Kim B-R, et al: Characterization of White Matter Injury in a Rat Model of Chronic Cerebral Hypoperfusion. Stroke 2016, 47(2):542-547.

21. Schuff N, Matsumoto S, Kmiecik J, Studholme C, Du A, Ezekiel F, Miller BL, Kramer JH, Jagust WJ, Chui HC, Weiner MW: Cerebral blood flow in ischemic vascular dementia and Alzheimer's disease, measured by arterial spin-labeling magnetic resonance imaging. Alzheimers \& Dementia 2009, 5:454-462.

22. Hattori Y, Enmi J-i, Iguchi S, Saito S, Yamamoto Y, Nagatsuka K, Iida H, Ihara M: Substantial Reduction of Parenchymal Cerebral Blood Flow in Mice with Bilateral Common Carotid Artery Stenosis. Scientific Reports 2016, 18(6):32179.

23. Adams D, Cheng F, Jou H, Aung S, Yasui Y, Vohra S: The Safety of Pediatric Acupuncture: A Systematic Review. Pediatrics 2011, 128:E1575-E1587.

24. Shi G-X, Liu C-Z, Guan W, Wang Z-Z, Wang L, Xiao C, Li Z-G, Li Q-Q, 
Wang L-P: Effects of acupuncture on Chinese medicine syndromes of vascular dementia. Chinese Journal of Integrative Medicine 2014, 20:661-666.

25. Yu J-C, Zhang X-Z, Liu C-Z, Meng Y-C, Han J-X: Effect of acupuncture treatment on vascular dementia. Neurological Research 2006, 28:97-103.

26. Uchida S, Kagitani F: Effect of acupuncture-like stimulation on cortical cerebral blood flow in aged rats. Journal of Physiological Sciences 2015, $65: 67-75$.

27. Zhang X, Wu B, Nie K, Jia Y, Yu J: Effects of acupuncture on declined cerebral blood flow, impaired mitochondrial respiratory function and oxidative stress in multi-infarct dementia rats. Neurochemistry International 2014, 65:23-29.

28. Ye Y, Li H, Yang J-W, Wang X-R, Shi G-X, Yan C-Q, Ma S-M, Zhu W, Li Q-Q, Li T-R, et al: Acupuncture Attenuated Vascular Dementia-Induced Hippocampal Long-Term Potentiation Impairments via Activation of D1/D5 Receptors. Stroke 2017, 48:1044-1051.

29. Basser PJ, Mattiello J, Lebihan D: ESTIMATION OF THE EFFECTIVE SELF-DIFFUSION TENSOR FROM THE NMR SPIN-ECHO. Journal of Magnetic Resonance Series B 1994, 103:247-254.

30. CW GP: The rat brain in stereotaxic coordinates. 2nd editon. Academic Press; San diego. 1996.

31. Fernando MS, Simpson JE, Matthews F, Brayne C, Lewis CE, Barber R, Kalaria RN, Forster G, Esteves F, Wharton SB, et al: White matter lesions in an unselected cohort of the elderly: molecular pathology suggests origin from chronic hypoperfusion injury. Stroke 2006, 37:1391-1398.

32. Ding N, Jiang J, Xu A, Tang Y, Li Z: Manual Acupuncture Regulates Behavior and Cerebral Blood Flow in the SAMP8 Mouse Model of Alzheimer's Disease. Front Neurosci 2019, 13:37.

33. Wang X-R, Shi G-X, Yang J-W, Yan C-Q, Lin L-T, Du S-Q, Zhu W, He T, Zeng X-H, Xu Q, Liu CZ: Acupuncture ameliorates cognitive impairment and hippocampus neuronal loss in experimental vascular dementia through Nrf2-mediated antioxidant response. Free Radic Biol Med 2015, 89:1077-1084.

34. Xiao L-Y, Wang X-R, Yang J-W, Ye Y, Zhu W, Cao Y, Ma S-M, Liu C-Z: 
Acupuncture Prevents the Impairment of Hippocampal LTP Through beta1-AR in Vascular Dementia Rats. Mol Neurobiol 2018, 55:7677-7690.

35. Ye Y, Li H, Yang J-W, Wang X-R, Shi G-X, Yan C-Q, Ma S-M, Zhu W, Li Q-Q, Li T-R, et al: Acupuncture Attenuated Vascular Dementia-Induced Hippocampal Long-Term Potentiation Impairments via Activation of D1/D5 Receptors. Stroke 2017, 48:1044-1051.

36. Alfaro FJ, Gavrieli A, Saade-Lemus P, Lioutas VA, Upadhyay J, Novak V: White matter microstructure and cognitive decline in metabolic syndrome: a review of diffusion tensor imaging. Metabolism 2018, 78:52-68.

37. Kim Y-J, Kwon H-K, Lee J-M, Kim Y-J, Kim H-J, Jung N-Y, Kim S-T, Lee K-H, Na D-L, Seo S-W: White matter microstructural changes in pure Alzheimer's disease and subcortical vascular dementia. Eur J Neurol 2015, 22:709-716.

38. Wang X, Lin F, Gao Y, Lei H: Bilateral common carotid artery occlusion induced brain lesions in rats: A longitudinal diffusion tensor imaging study. Magn Reson Imaging 2015, 33:551-558.

39. Saggu R, Schumacher T, Gerich F, Rakers C, Tai K, Delekate A, Petzold GC: Astroglial NF-kB contributes to white matter damage and cognitive impairment in a mouse model of vascular dementia. Acta Neuropathologica Communications 2016, 4(1):76. 


\section{Figure legends}

\section{Figure 1. Experiments procedures}

BCCAO: bilateral common carotid arteries occlusion.

Figure 2. Acupuncture ameliorates spatial and learning memory of VaD rats.

A. Escape latency and B. Swimming speed in training, and C. The percentage of time spent in the target quadrant in probe trial were measured in SHAM, BCCAO, ACU, and NON-ACU groups by using Morris water maze. Data are presented as mean \pm SEM ( $\mathrm{n}=6$ per group). $* * \mathrm{p}<0.01, * * * \mathrm{p}<0.001, * * * * \mathrm{p}<0.0001$, respectively, compared with SHAM group; \& $\mathrm{p}<0.05, \& \& \mathrm{p}<0.01$, \&\&\& $\mathrm{p}<0.001$, respectively, compared with BCCAO group. SHAM: sham-operated group, BCCAO: BCCAO-operated group, ACU: BCCAO-operated + acupuncture at GV20 and ST36 group, NON-ACU: BCCAO-operated + acupuncture at non-acupoints group.

\section{Figure 3. Acupuncture improves white matter perfusion of VaD rats.}

A. Reconstructed cerebral blood flow map, and the white matter ROIs (red dotted line). B. the quantification of cerebral blood flow of whiter matter regions by arterial spin labeling. Data are presented as mean \pm SEM ( $n=8$ per group). $* * p<0.01$. ROIs: regions of interest, $\mathrm{ON}$ : optic nerve, $\mathrm{CC}$ : corpus callosum, EC: external capsule, IC: internal capsule, OT: optic tract, SHAM: sham-operated group, BCCAO: BCCAO-operated group, ACU: BCCAO-operated + acupuncture at GV20 and ST36 group, NON-ACU: BCCAO-operated + acupuncture at non-acupoints group.

Figure 4. Acupuncture protects white matter integrity from cerebral chronic hypoperfusion of VaD rats.

A. The white matter ROIs drawn from T2WI image of rat brain. B. Quantification of DTI parameters in the white matter ROIs in SHAM, BCCAO, ACU, and NON-ACU groups by using diffusion tensor imaging. C. In corpus callosum, the two-tail Pearson's correlation analysis revealed significant positive correlation between CBF and FA, and negative correlation between CBF and RD. Values are presented as mean \pm SEM (n=8 per group). * $\mathrm{p}<0.05, * * \mathrm{p}<0.01, * * * \mathrm{p}<0.001, * * * * \mathrm{p}<0.0001$, respectively. ROIs: regions of interest, FA: fractional anisotropy, RD: radial diffusivity, $\mathrm{AD}$ : axial diffusivity, $\mathrm{MD}$ : mean diffusivity, $\mathrm{CC}$ : corpus callosum, $\mathrm{EC}$ : external capsule, IC: internal capsule, ON: optic nerve, OT: optic tract, CBF: cerebral blood flow, SHAM: sham-operated group, BCCAO: BCCAO-operated group, ACU: BCCAO-operated + acupuncture at GV20 and ST36 group, NON-ACU: 
BCCAO-operated + acupuncture at non-acupoints group.

Figure 5. Visualization and quantification of white matter tracts indices in rats.

A. Visualization of corpus collosum-associated white matter ROIs by reconstructing DTI images. B. quantification of whiter matter fiber density (fiber number/voxel) and fiber length in SHAM, BCCAO, ACU, and NON-ACU groups. Data are presented as mean \pm SEM ( $n=8$ per group). C. In corpus callosum, the two-tail Pearson's correlation analysis revealed significant positive correlation between $\mathrm{CBF}$ and fiber density. ${ }^{* *} \mathrm{p}<0.01$. ROIs: regions of interest, SHAM: sham-operated group, BCCAO: BCCAO-operated group, ACU: BCCAO-operated + acupuncture at GV20 and ST36 group, NON-ACU: BCCAO-operated + acupuncture at non-acupoints group.

Figure 6. Acupuncture attenuates loss of myelin sheath and microglia activation in VaD rats.

A. Immunostaining of $\mathrm{MBP}, \mathrm{Iba}-1^{+}$and $\mathrm{GFAP}^{+}$glia, and B. Calculations of $\mathrm{MBP}^{+}$ area, Iba- $1^{+}$cell number and $\mathrm{GFAP}^{+}$cell number in white matter of SHAM, BCCAO, ACU, and NON-ACU groups. Scale bar: $50 \mu \mathrm{m}$, data are presented as mean \pm SEM ( $\mathrm{n}=6$ per group). $* * \mathrm{p}<0.01, * * * \mathrm{p}<0.001$. MBP: myelin basic protein, Iba-1: ionized calcium binding adapter molecule 1, GFAP: glial fibrillary acidic protein, SHAM: sham-operated group, BCCAO: BCCAO-operated group, ACU: BCCAO-operated + acupuncture at GV20 and ST36 group, NON-ACU: BCCAO-operated + acupuncture at non-acupoints group. 